\title{
Minimizing Energy Cost in Electric Arc Furnace Steel Making by Optimal Control Designs
}

\author{
Er-wei Bai ${ }^{1,2}$ \\ ${ }^{1}$ Department of Electrical and Computer Engineering, University of Iowa, Iowa City, LA 52242, USA \\ ${ }^{2}$ School of Electronics, Electrical Engineering and Computer Science, Queen's University, Belfast BT7 INN, UK \\ Correspondence should be addressed to Er-wei Bai; er-wei-bai@uiowa.edu \\ Received 30 September 2013; Revised 23 December 2013; Accepted 14 January 2014; Published 20 February 2014 \\ Academic Editor: Qilian Liang \\ Copyright $\odot 2014$ Er-wei Bai. This is an open access article distributed under the Creative Commons Attribution License, which \\ permits unrestricted use, distribution, and reproduction in any medium, provided the original work is properly cited.

\begin{abstract}
Production cost in steel industry is a challenge issue and energy optimization is an important part. This paper proposes an optimal control design aiming at minimizing the production cost of the electric arc furnace steel making. In particular, it is shown that with the structure of an electric arc furnace, the production cost which is a linear programming problem can be solved by the tools of linear quadratic regulation control design that not only provides an optimal solution but also is in a feedback form. Modeling and control designs are validated by the actual production data sets.
\end{abstract}

\section{Introduction}

Steel industries recycle scrap steel using the electric arc furnace (EAF) by melting it and changing its chemical composition to produce different product grades. Obviously, steel industry is one of the greatest energy consuming sectors and there is a strong demand to decrease the use of electricity and other forms of energy in the EAF steel making.

In general, the current melting process control is manual and the production cost is not optimal. Steel industries have some nominal automation for EAFs but are mostly operator driven. Although operator intuition is invaluable for such industries, it rests on the recipes that have been effective in the past and becomes difficult to take into account all possible uncertainties unless a mathematical framework is developed for the system and an automation process is in place. It has been shown that a substantial part of the energy consumption is wasted in melting the scrap [1-3]. This illustrates that there is a tremendous opportunity for control to play.

Unlike most existing papers in the literature, this paper concerns the production cost which is related to energy consumption for melting process and the way to achieve the optimality. Therefore, the purpose of the model is different from the large part of the existing literature [1-6]. For example, in [1], an adaptive model predictive control was proposed to follow the preset trajectories. How to design the preset trajectories was not discussed. In [2], a PID controller was proposed to have the electrodes maintain constant power consumption. In [3], a model was developed but there was no discussion on how to control it. In [6], a model was proposed to achieving the maximum power input to the melting process. In all these works, minimizing control consumption was not a concern. In the work reported here, a mathematical model of EAFs is developed and its unknown parameters are then estimated. The goal of the model is to design a control algorithm that minimizes the energy consumption. The model adopted in this paper is a linear time invariant system which seems to work satisfactorily. More importantly, in the second step, optimal control inputs are calculated aiming at minimizing the production cost. Because the actual production cost is linear in the consumptions of energy and graphite electrode, the optimization is a linear programming problem. A problem of the linear programming is that it provides an open loop input design. Though optimal in the absence of model uncertainty and measurement noise, its performance in reality cannot be guaranteed under inevitable model uncertainty and measurement noise. The contribution of the paper is to show that with the particular structure of the EAF, the linear programming problem can in fact be solved by the well-known linear quadratic regulation (LQR) problem 


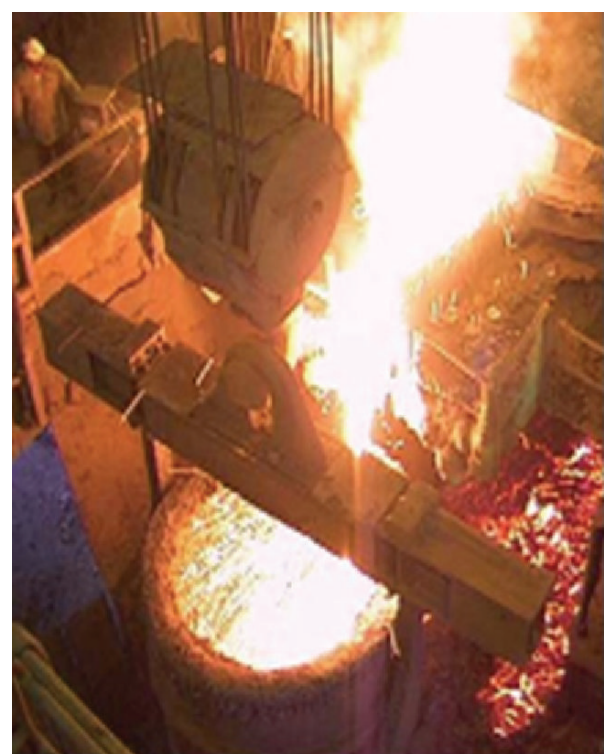

FIgURe 1: Electric arc furnace.

which is in a feedback form and thus is more robust than an open loop solution.

All the data sets used in this study for modeling and control validation are actual production data collected from the Gerdau Ameristeel mill facility in Wilton Iowa that is composed of a scrap shredding facility, an electric arc furnace (EAF) as shown in Figure 1, several continuous billet casters, and a rolling mill. Because of proprietary properties, no actual data value is revealed in the paper, but the modeling results and final performance improvements are shown.

\section{Problem Statement}

In this section, we describe the system model and the goal of optimal control. Clearly, EAF models could be different for different purposes. Our goal is to optimize the production cost while the quality and efficiency are maintained. To this end, we focus on 7 key variables that provide a fairly reasonable description of the dynamics of an EAF:

(i) kilowatt-hour consumption (KWH),

(ii) electrode consumption $\left(I^{2} h\right)$,

(iii) percentage of scrap melted (PM),

(iv) average arc current $\left(I_{\text {avg }}\right)$,

(v) oxygen input $\left(\mathrm{O}_{2}\right)$,

(vi) gas input (Gas),

(vii) carbon input (Car).

All 7 variables are either measurable or computable in real time. Among these variables, the kilowatt-hour consumption $(\mathrm{KWH})$ is the consumption of electrical energy that constitutes a substantial part of the total cost, the electrode consumption $\left(I^{2} h\right)$ is the consumption of the graphite electrode that also contributes to the cost significantly, and the percentage of scrape melted (PM) is the percentage of scrap melted which is a quality constraint on the melting control. At the end of each melting process, PM has to be $100(\%)$. Other 4 variables are electrical energy input $I_{\text {avg }}$ and chemical energy inputs $\mathrm{O}_{2}$ (oxygen), Gas (gas), and Car (carbon), respectively. All 4 energy inputs contribute to the total cost and among them $I_{\text {avg }}$ is the most expensive one.

Based on the physics, the increments of the kilowatt-hour consumption $(\mathrm{KWH})$ and the electrode consumption $\left(I^{2} h\right)$ from time $k \delta t$ to $(k+1) \delta t$ are directly related to the current $I_{\text {avg }}$ in the interval $[k \delta t,(k+1) \delta t)$. On the other hand, the percentage of scrape melted (PM) is related to all energy inputs during the same period including the electrical energy as well as chemical energy. The EAF assumes the following structure:

$$
\begin{aligned}
&\left(\begin{array}{c}
\mathrm{KWH}[k+1] \\
I^{2} h[k+1] \\
\mathrm{PM}[k+1]
\end{array}\right)= \underbrace{\left(\begin{array}{lll}
1 & 0 & 0 \\
0 & 1 & 0 \\
0 & 0 & 1
\end{array}\right)}_{A}\left(\begin{array}{c}
\mathrm{KWH}[k] \\
I^{2} h[k] \\
\operatorname{PM}[k]
\end{array}\right) \\
& \underbrace{\left(\begin{array}{cccc}
\varphi_{1} & 0 & 0 & 0 \\
\phi_{1} & 0 & 0 & 0 \\
a_{1} & a_{2} & a_{3} & a_{4}
\end{array}\right)}_{B}\left(\begin{array}{c}
I_{\mathrm{avg}}[k] \\
\mathrm{O}_{2}[k] \\
\operatorname{Gas}[k] \\
\operatorname{Car}[k]
\end{array}\right), \\
& k=1, \ldots, N,
\end{aligned}
$$

where $\left(\begin{array}{c}\mathrm{KWH}[0] \\ I^{2} h[0] \\ \mathrm{PM}[0]\end{array}\right)=0, k=k \delta t$ with $\delta t=5(\mathrm{sec})$ being the sampling interval and $(N+1) \delta t$ is the total time by which $100 \%$ of scrap should be melted. This model is not surprising and has been used in the literature. To maintain the same productivity and efficiency, $(N+1) \delta$ is the total length of the melting process time and is predetermined by the current practice without optimal control. $B$ is a matrix whose entries depend on a particular EAF and the scrap loads. Note that $I_{\text {avg }}[k], \mathrm{O}_{2}[k]$, Gas $[k], \operatorname{Car}[k]$ are the respective total energy delivered to the EAF during the period $[k \delta t,(k+1) \delta t)$ and $\mathrm{KWH}[k]$ and $I^{2} h[k]$ are the total electric energy and electrode consumption, respectively, at the end of the $k$ th sampling interval. The production cost is defined as

$$
\begin{aligned}
J= & q_{1} \operatorname{KWH}[N+1]+q_{2} I^{2} h[N+1]+q_{3} \sum_{k=1}^{N} \mathrm{O}_{2}[k] \\
& +q_{4} \sum_{k=1}^{N} \operatorname{Car}[k]+q_{5} \sum_{k=1}^{N} \operatorname{Gas}[k],
\end{aligned}
$$

where $q_{1}, q_{2}, q_{3}, q_{4}$, and $q_{5}$ are the per unit cost of electric energy, electrode consumption, and the chemical energy of oxygen, gas, and carbon, respectively. The unit cost $q_{i}$ s fluctuate according to the market value and are adjusted when the market price changes. The goal of control is to design an input sequence $I_{\text {avg }}[k], \mathrm{O}_{2}[k], \operatorname{Gas}[k], \operatorname{Car}[k], k=1,2, \ldots, N$ so that the above cost function (2) is minimized under the constraint

$$
\operatorname{PM}(N+1)=100 .
$$


In addition, because of hardware constraints, the input energy must satisfy for each $k=1,2, \ldots, N$

$$
\begin{aligned}
& 0<I_{l} \leq I_{\mathrm{avg}}[k] \leq I_{u}, \\
& 0 \leq \mathrm{O}_{2}[k] \leq O_{u}, \\
& 0 \leq \operatorname{Gas}[k] \leq G_{u}, \\
& 0 \leq \operatorname{Car}[k] \leq C_{u},
\end{aligned}
$$

where $I_{l}, I_{u}, O_{u}, G_{u}$, and $C_{u}$ denote the physical bounds on the amount of electrical and chemical energy, respectively, that can be delivered to the EAF during each sampling interval $\delta t$.

\section{Optimal Control Calculation}

To simplify notation, in the rest of the paper, we let

$$
\begin{aligned}
& x[k]=\left(\begin{array}{l}
x_{1}[k] \\
x_{2}[k] \\
x_{3}[k]
\end{array}\right)=\left(\begin{array}{c}
\mathrm{KWH}[k] \\
I^{2} h[k] \\
\mathrm{PM}[k]
\end{array}\right), \\
& u[k]=\left(\begin{array}{l}
u_{1}[k] \\
u_{2}[k] \\
u_{3}[k] \\
u_{4}[k]
\end{array}\right)=\left(\begin{array}{c}
I_{\mathrm{ave}}[k] \\
\mathrm{O}_{2}[k] \\
\mathrm{Gas}[k] \\
\mathrm{Car}[k]
\end{array}\right) .
\end{aligned}
$$

Further, by some simple calculations, we notice that

$$
\begin{gathered}
\mathrm{KWH}[N+1]=\varphi_{1} \sum_{k=1}^{N} I_{\mathrm{avg}}[k], \\
I^{2} h[N+1]=\phi_{1} \sum_{k=1}^{N} I_{\mathrm{avg}}[k], \\
\mathrm{PM}[N+1]=a_{1} \sum_{k=1}^{N} I_{\mathrm{avg}}[k]+a_{2} \sum_{k=1}^{N} \mathrm{O}_{2}[k] \\
+a_{3} \sum_{k=1}^{N} \operatorname{Gas}[k]+a_{4} \sum_{k=1}^{N} \operatorname{Car}[k] .
\end{gathered}
$$

Therefore, the cost function of (2) can be rewritten as

$$
\begin{aligned}
J= & p_{1} \sum_{k=1}^{N} I_{\mathrm{avg}}[k]+p_{2} \sum_{k=1}^{N} \mathrm{O}_{2}[k] \\
& +p_{3} \sum_{k=1}^{N} \operatorname{Car}[k]+p_{4} \sum_{k=1}^{N} \operatorname{Gas}[k],
\end{aligned}
$$

where $p_{1}=q_{1} \varphi_{1}+q_{2} \phi_{1}, p_{2}=q_{3}, p_{3}=q_{4}$, and $p_{4}=q_{5}$. To recap, the goal is to design an input sequence $u_{i}[k], i=$ $1,2,3,4, k=1,2, \ldots, N$ for a given $N$ so that the cost function

$$
\begin{aligned}
J= & p_{1} \sum_{k=1}^{N} u_{1}[k]+p_{2} \sum_{k=1}^{N} u_{2}[k]+p_{3} \sum_{k=1}^{N} u_{3}[k] \\
& +p_{4} \sum_{k=1}^{N} u_{4}[k]
\end{aligned}
$$

is minimized under the equality and inequality constraints,

$$
\begin{aligned}
x[k+1]= & \underbrace{\left(\begin{array}{lll}
1 & 0 & 0 \\
0 & 1 & 0 \\
0 & 0 & 1
\end{array}\right) x[k]}_{A} \\
& +\underbrace{\left(\begin{array}{cccc}
\varphi_{1} & 0 & 0 & 0 \\
\phi_{1} & 0 & 0 & 0 \\
\theta_{1} & \theta_{2} & \theta_{3} & \theta_{4}
\end{array}\right)}_{B} u[k], \\
& x[0]=0, \quad k=1,2, \ldots, N, \\
0< & I_{l} \leq u_{1}[k] \leq I_{u}, \\
& \leq \leq u_{2}[k] \leq O_{u}, \\
& 0 \leq u_{3}[k] \leq G_{u}, \\
& 0 \leq u_{4}[k] \leq C_{u}, \\
100= & a_{1} \sum_{k=1}^{N} u_{1}[k]+a_{2} \sum_{k=1}^{N} u_{2}[k] \\
+ & a_{3} \sum_{k=1}^{N} u_{3}[k]+a_{4} \sum_{k=1}^{N} u_{4}[k] .
\end{aligned}
$$

Here, KWH, $I^{2} h$, and PM describe the 3-dimensional state space and $I_{\text {avg }}, \mathrm{O}_{2}, \mathrm{Car}$, and Gas describe the 4-dimensional input space. Matrices $A$ and $B$ are given as before.

3.1. Solution Using Linear Programming. The optimal control design problem described above is exactly a linear programming problem with a linear objective function and linear equality and inequality constraints. This can be solved by commercially available software packages. For real time applications, one issue is its computational complexity. In reality, $N$ is approximately 1200 which implies that there are $3 \cdot 1200=3600$ state variables, $4 \cdot 1200=4800$ input variables, $2 \cdot 4 \cdot 1200=9600$ inequality constraints, and $3 \cdot 1200=3600$ equality constraints. Simply put, it is a linear programming problem with 8400 variables and 14400 constraints. The linear programming has to be recalculated every time when the model experiences some changes. The computational power required for a real time application is not favorable. The second problem is that the linear programming provides an open loop control. Yes, the solution $u^{*}[k], k=1,2, \ldots, N$ of the linear programming is optimal if everything is ideal. In reality, model uncertainty and measurement noise are always present and the linear programming solution is open loop. It is impossible to guarantee a good performance of an open loop control system in the presence of uncertainty and noise. What we prefer is a solution that minimizes the cost function (8) under the constraints (9) but in a closed loop form. To this end, note that the linear quadratic regulation (LQR) control design has been extensively studied in the literature. The solution of LQR is in a feedback form which is more robust than the open loop solution provided by the linear programming. Moreover, the LQR control has many desirable 
properties. We will show in the next section that by proper choices of the weights in the LQR calculation, the solution of the LQR also solves the linear programming problem or equivalently the cost function (8) under constraints (9) but in a closed loop form.

Though the linear programming solution may not be practically useful because of its open loop nature, it does reveal some important insights. Let

$$
U_{i}=\sum_{k=1}^{N} u_{i}[k], \quad i=1,2,3,4 .
$$

Then, the cost function and the terminal constraint can be rewritten, respectively, as

$$
\begin{gathered}
(J=) J_{\mathrm{LP}}=p_{1} U_{1}+p_{2} U_{2}+p_{3} U_{3}+p_{4} U_{4}, \\
100=a_{1} U_{1}+a_{2} U_{2}+a_{3} U_{3}+a_{4} U_{4} .
\end{gathered}
$$

Also from the state space equation, we have $x_{1}[N+1]=\varphi_{1} U_{1}$ and $x_{2}[N+1]=\phi U_{1}$. Now suppose $u_{i}^{*}[k]$ 's, $k=1,2, \ldots, N$, $i=1,2,3,4$ are the solution of the linear programming. Define

$$
\begin{gathered}
u_{i}[k]=\frac{1}{N} \sum_{m=1}^{N} u_{i}^{*}[m]=\frac{1}{N} U_{i}, \\
i=1,2,3,4, k=1,2, \ldots, N .
\end{gathered}
$$

Clearly, $\sum_{k=1}^{N} u_{i}[k]=\sum_{k=1}^{N} u_{i}^{*}[k]=U_{i}$ and $\min _{m} u_{i}^{*}[m] \leq$ $u_{i}[k] \leq \max _{m} u_{i}^{*}[m]$ for each $i=1,2,3,4$ and every $k=1,2, \ldots, N$. Therefore, the solutions $u_{i}[k]$ 's of (12) are constants that minimize the cost function (8) and satisfy all the equality and inequality constraints (9). In short, the solution of the linear programming may not be unique, but there always exists a constant solution. We summarize this observation in a theorem form that will be used later.

Theorem 1. Consider the optimal control problem of (8) under the constraints (9). Then, there always exist constant sequences $u_{i}[k] \equiv u_{i}[m]$ 's, $i=1,2,3,4$, and $1 \leq k, m \leq N$ that solve the problem.

3.2. LQR Design. Linear quadratic regulator problem is extensively studied in the literature and its solution can be readily found in a number of textbooks $[7,8]$. In addition, its properties in terms of stability, robustness, and so forth, are well documented and in fact are favorable [7, 8]. The difference is however that LQR minimizes a quadratic performance function

$$
\begin{aligned}
J_{\mathrm{LQR}}= & x[N+1]^{T} S x[N+1] \\
& +\sum_{k=1}^{N}\left(x[k]^{T} \mathrm{Q} x[k]+u[k]^{T} R u[k]\right),
\end{aligned}
$$

where $S=S^{T} \geq 0, Q=Q^{T} \geq 0$, and $R=R^{T}>0$ are the terminal state, state and input cost matrices, respectively. Our goal in this section is to show that by some properly chosen matrixes $S, Q$, and $R$, the LQR solution also solves the linear programming problem which is the goal of the control design. It is emphasized that the LQR is not only more robust but also numerically efficient. Note that the LQR is recursive and at each time $k t$, only a fixed dimension Riccati equation (in our case, 4 dimensional) needs to be calculated $[7,8]$.

To this end, we set

$$
S=0, \quad Q=0, \quad R=\left(\begin{array}{cccc}
r_{1} & 0 & 0 & 0 \\
0 & r_{2} & 0 & 0 \\
0 & 0 & r_{3} & 0 \\
0 & 0 & 0 & r_{4}
\end{array}\right)
$$

for some $r_{i}>0$ or equivalently

$$
\begin{aligned}
J_{\mathrm{LQR}}\left(u\left(r_{1}, r_{2}, r_{3}, r_{4}\right)\right)= & \sum_{k=1}^{N} u[k]^{T} R u[k] \\
= & r_{1} \sum_{k=1}^{N} u_{1}^{2}[k]+r_{2} \sum_{k=1}^{N} u_{2}^{2}[k] \\
& +r_{3} \sum_{k=1}^{N} u_{3}^{2}[k]+r_{4} \sum_{k=1}^{N} u_{4}^{2}[k],
\end{aligned}
$$

where $J_{\mathrm{LQR}}\left(u\left(r_{1}, r_{2}, r_{3}, r_{4}\right)\right)$ indicates that the cost depends on the optimal input sequence $u[k]$ which in turn depends on the weights $r_{i}$ 's. Together with the terminal constraint in (9), the LQR solution assumes the form of $[7,8]$

$$
u[k]=K[k] x[k]+H[k] x_{3}[N+1]
$$

where $K[\cdot]$ and $H[\cdot]$ are functions of $r_{1}, r_{2}, r_{3}, r_{4}$. Note that the control sequence is in a feedback form. Interested readers may find more details in many textbooks, for example, $[7,8]$. It is easy to verify the following theorem.

Theorem 2. Consider the above LQR problem of (15) under the constraints (9). Then, the input sequences $u_{i}^{*}[k]$ 's, $i=$ $1,2,3,4, k=1,2, \ldots, N$ that solve the LQR problem must be constant sequences $u_{i}^{*}[k] \equiv u_{i}^{*}[m]$ for all $i=1,2,3,4$ and $1 \leq k, m \leq N$.

Proof. The proof is by contradiction. Suppose not, there exists at least one $k$ so that $u_{i}^{*}[k] \neq u_{i}^{*}[k+1]$ for some $i$. Let $u_{i}[m]$ 's, $i=1,2,3,4$, and $m=1,2, \ldots, N$ be the exact sequences of $u_{i}^{*}[m]$ except at $k$ and $k+1$,

$$
u_{i}[k]=u_{i}[k+1]=\frac{1}{2}\left(u_{i}^{*}[k]+u_{i}^{*}[k+1]\right) .
$$


Then,

$$
\begin{gathered}
r_{i}\left(u_{i}^{2}[k]+u_{i}^{2}[k+1]\right) \\
=r_{i}\left(\left(\frac{\left(u_{i}^{*}[k]+u_{i}^{*}[k+1]\right)}{2}\right)^{2}\right. \\
\left.\quad+\left(\frac{\left(u_{i}^{*}[k]+u_{i}^{*}[k+1]\right)}{2}\right)^{2}\right) \\
=r_{i} \frac{\left(u_{i}^{*}[k]+u_{i}^{*}[k+1]\right)^{2}}{2} \\
<r_{i}\left(u_{i}^{* 2}[k]+u_{i}^{* 2}[k+1]\right) .
\end{gathered}
$$

In other words, all the constraints of (9) are satisfied by the new sequences $u_{i}[k]$, but the cost function generated by $u_{i}[k]$ is strictly smaller than the cost function generated by $u_{i}^{*}[k]$. Therefore, the optimal sequences have to be constant sequences. This finishes the proof.

Now let the solution sequence of the LQR problem (15) under the constraints (9) be denoted by

$$
\begin{array}{r}
u_{1}^{\mathrm{LQR}}\left(r_{i}\right), u_{2}^{\mathrm{LQR}}\left(r_{i}\right), u_{3}^{\mathrm{LQR}}\left(r_{i}\right), u_{4}^{\mathrm{LQR}}\left(r_{i}\right), \\
i=1,2,3,4, \quad k=1,2, \ldots, N .
\end{array}
$$

Further, let

$$
\left(r_{1}^{*}, r_{2}^{*}, r_{3}^{*}, r_{4}^{*}\right)=\arg \min _{r_{i}} J_{\mathrm{LQR}}\left(u\left(r_{1}, r_{2}, r_{3}, r_{4}\right)\right) .
$$

Now we claim the following.

Theorem 3. The solution of the LQR problem $u_{i}^{L Q R}\left(r_{1}^{*}, r_{2}^{*}, r_{3}^{*}, r_{4}^{*}\right)^{\prime} s, \quad i=1,2,3,4$, defined above also solves the linear programming problem of (8) and (9).

Proof. Since both the linear programming problem and the LQR problem assume constant solutions, the goal is to find constant $u_{i}$ 's for all $k$ that solve, respectively, the linear programming problem and the LQR problem as shown in Table 1.

For the linear programming problem, at least one of the optimal solution lies at one of the corners formed by the intersections of the hyperplanes of the cost function, the terminal constraint, and the inequality constraints as shown in Figure 2 for a two-dimension case. On the other hand, the solution of the LQR problem is at the intersection of the same hyperplanes formed by the terminal and inequality constraints and an ellipsoid

$$
\begin{aligned}
1= & \frac{\left(u_{1}^{\mathrm{LQR}}\left(r_{i}\right)\right)^{2}}{\left(\sqrt{J_{\mathrm{LQR}} / r_{1} N}\right)^{2}}+\frac{\left(u_{2}^{\mathrm{LQR}}\left(r_{i}\right)\right)^{2}}{\left(\sqrt{J_{\mathrm{LQR}} / r_{2} N}\right)^{2}} \\
& +\frac{\left(u_{3}^{\mathrm{LQR}}\left(r_{i}\right)\right)^{2}}{\left(\sqrt{J_{\mathrm{LQR}} / r_{3} N}\right)^{2}}+\frac{\left(u_{4}^{\mathrm{LQR}}\left(r_{i}\right)\right)^{2}}{\left(\sqrt{J_{\mathrm{LQR}} / r_{4} N}\right)^{2}}
\end{aligned}
$$

TABLE 1

\begin{tabular}{cc}
\hline LP & $\mathrm{LQR}$ \\
\hline $\min _{u_{i}} J_{\mathrm{LP}}\left(u_{i}\right)=N \sum_{i=1}^{4} p_{i} u_{i}$ & $\min _{u_{i}} J_{\mathrm{LQR}}\left(u_{i}\left(r_{1}, r_{2}, r_{3}, r_{4}\right)\right)=$ \\
& $N \sum_{i=1}^{4} r_{i} u_{i}^{2}\left(r_{1}, r_{2}, r_{3}, r_{4}\right)$ \\
$x_{1}[N+1]=N \varphi_{1} u_{1}$ & $x_{1}[N+1]=N \varphi_{1} u_{1}\left(r_{i}\right)$ \\
s.t. $\quad x_{2}[N+1]=N \phi_{1} u_{1}$ & $x_{2}[N+1]=N \phi_{1} u_{1}\left(r_{i}\right)$ \\
$x_{3}[N+1]=N \sum_{i=1} a_{i} u_{i}$ & $x_{3}[N+1]=N \sum_{i=1} a_{i} u_{i}\left(r_{1}, r_{2}, r_{3}, r_{4}\right)$ \\
$0<I_{l} \leq u_{1} \leq I_{u}$ & $0<I_{l} \leq u_{1}\left(r_{i}\right) \leq I_{u}$ \\
$0 \leq u_{2} \leq O_{u}$ & $0 \leq u_{2}\left(r_{i}\right) \leq O_{u}$ \\
$0 \leq u_{3} \leq G_{u}$ & $0 \leq u_{3}\left(r_{i}\right) \leq G_{u}$ \\
$0 \leq u_{4} \leq C_{u}$ & $0 \leq u_{4}\left(r_{i}\right) \leq C_{u}$
\end{tabular}

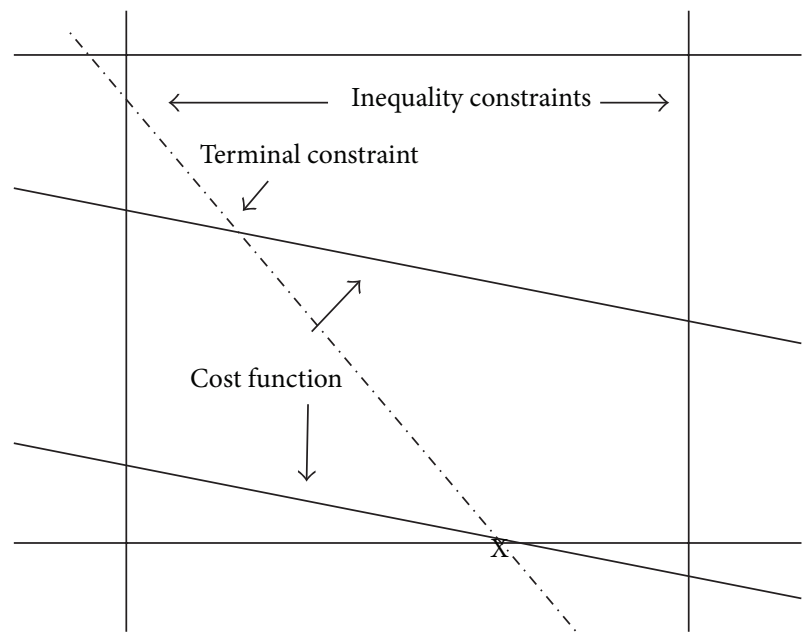

FIGURE 2: $X=$ solution of linear programming.

for a given set of $r_{i}$. By varying $r_{i}$, the axes of the ellipsoid can be adjusted arbitrarily and there always exist some $r_{i}^{*}$ 's so that the LQR solution is at one of the corners that is also a solution of the linear programming problem as illustrated in Figure 3 for a two-dimensional case. Denote such solutions by $u_{1}^{\mathrm{LQR}}\left(r_{i}^{*}\right), u_{2}^{\mathrm{LQR}}\left(r_{i}^{*}\right), u_{3}^{\mathrm{LQR}}\left(r_{i}^{*}\right), u_{4}^{\mathrm{LQR}}\left(r_{i}^{*}\right)$. This implies

$$
\begin{gathered}
J_{\mathrm{LP}}\left(u_{1}^{\mathrm{LQR}}\left(r_{i}^{*}\right), u_{2}^{\mathrm{LQR}}\left(r_{i}^{*}\right), u_{3}^{\mathrm{LQR}}\left(r_{i}^{*}\right), u_{4}^{\mathrm{LQR}}\left(r_{i}^{*}\right)\right) \\
\quad \leq J_{\mathrm{LP}}\left(u_{1}, u_{2}, u_{3}, u_{4}\right)
\end{gathered}
$$

for any $u_{i}$ or the linear programming cost function $J_{\mathrm{LP}}$ evaluated at the LQR solution $u_{1}^{\mathrm{LQR}}\left(r_{i}^{*}\right), u_{2}^{\mathrm{LQR}}\left(r_{i}^{*}\right), u_{3}^{\mathrm{LQR}}\left(r_{i}^{*}\right)$, $u_{4}^{\mathrm{LQR}}\left(r_{i}^{*}\right)$ is no larger than $J_{\mathrm{LP}}\left(u_{1}, u_{2}, u_{3}, u_{4}\right)$ for any $u_{i}$. On the other hand,

$$
\begin{aligned}
\min _{u_{i}} & J_{\mathrm{LP}}\left(u_{i}\right) \\
& \leq J_{\mathrm{LP}}\left(u_{1}^{\mathrm{LQR}}\left(r_{i}^{*}\right), u_{2}^{\mathrm{LQR}}\left(r_{i}^{*}\right), u_{3}^{\mathrm{LQR}}\left(r_{i}^{*}\right), u_{4}^{\mathrm{LQR}}\left(r_{i}^{*}\right)\right) .
\end{aligned}
$$

Thus, the solution $u_{1}^{\mathrm{LQR}}\left(r_{i}^{*}\right), u_{2}^{\mathrm{LQR}}\left(r_{i}^{*}\right), u_{3}^{\mathrm{LQR}}\left(r_{i}^{*}\right), u_{4}^{\mathrm{LQR}}\left(r_{i}^{*}\right)$ of the LQR also solves the LP problem. This completes the proof. 


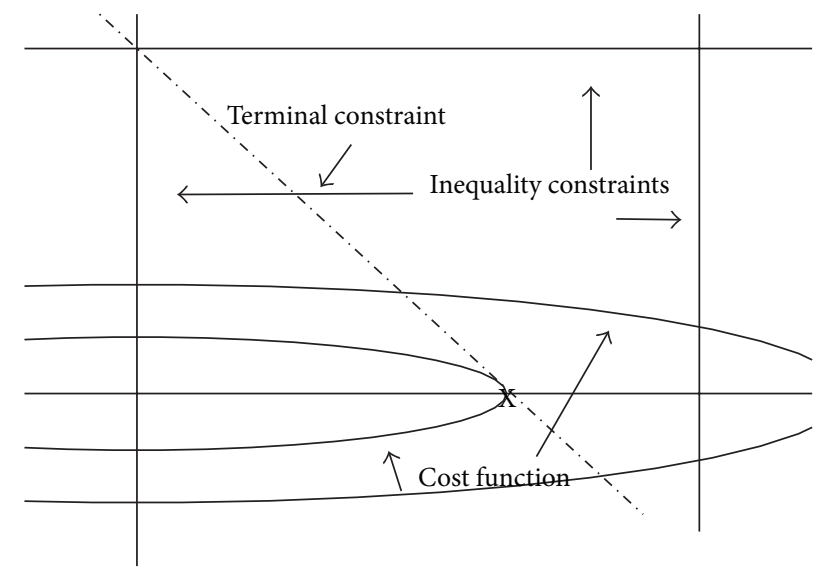

FIGURE 3: $X=$ solution of LQR.

3.3. Computation and Validation of $r_{i}^{*}$ 's. What the results of Theorem 3 say is that there exist constants $u_{i}$ 's that solve both the LQR and LP problems. Furthermore, let $u_{1}\left(r_{i}\right), u_{2}\left(r_{i}\right)$, $u_{3}\left(r_{i}\right), u_{4}\left(r_{i}\right)$ be the solution of LQR for given $r_{1}, r_{2}, r_{3}$, and $r_{4}$. Then,

$$
\left(r_{1}^{*}, r_{2}^{*}, r_{3}^{*}, r_{4}^{*}\right)=\arg \min _{r_{i}} J_{\mathrm{LP}}\left(u_{1}\left(r_{i}\right), u_{2}\left(r_{i}\right), u_{3}\left(r_{i}\right), u_{4}\left(r_{i}\right)\right)
$$

and this provides a way to compute $r_{i}^{*}$.

For simplicity, let us leave out all the inequality constraint in (9) first. Consider the terminal constraint and the cost function,

$$
\begin{aligned}
& J_{\mathrm{LQR}}=N\left(r_{1} u_{1}^{2}+r_{2} u_{2}^{2}+r_{3} u_{3}^{2}+r_{4} u_{4}^{2}\right), \\
& 100=N\left(a_{1} u_{1}+a_{2} u_{2}+a_{3} u_{3}+a_{4} u_{4}\right) .
\end{aligned}
$$

To find the LQR solution, we introduce the Lagrange multiplier

$$
\begin{aligned}
F= & N\left(r_{1} u_{1}^{2}+r_{2} u_{2}^{2}+r_{3} u_{3}^{2}+r_{4} u_{4}^{2}\right) \\
& +\lambda(100-N)\left(a_{1} u_{1}+a_{2} u_{2}+a_{3} u_{3}+a_{4} u_{4}\right) .
\end{aligned}
$$

Setting the derivatives of $F$ with respect to $u_{1}, u_{2}, u_{3}, u_{4}$ and $\lambda$ to zero implies that

$$
\begin{gathered}
u_{1}=\frac{\lambda a_{1}}{2 r_{1}}, \quad u_{2}=\frac{\lambda a_{2}}{2 r_{2}}, \\
u_{3}=\frac{\lambda a_{3}}{2 r_{3}}, \quad u_{4}=\frac{\lambda a_{4}}{2 r_{4}}, \\
\lambda=\frac{200}{N}\left[\frac{a_{1}^{2}}{r_{1}}+\frac{a_{2}^{2}}{r_{2}}+\frac{a_{3}^{2}}{r_{3}}+\frac{a_{4}^{2}}{r_{4}}\right]^{-1} .
\end{gathered}
$$

By solving these 5 equations, we have

$$
\begin{aligned}
& u_{1}=\frac{100 a_{1}}{r_{1} N}\left[\frac{a_{1}^{2}}{r_{1}}+\frac{a_{2}^{2}}{r_{2}}+\frac{a_{3}^{2}}{r_{3}}+\frac{a_{4}^{2}}{r_{4}}\right]^{-1}, \\
& u_{2}=\frac{100 a_{2}}{r_{2} N}\left[\frac{a_{1}^{2}}{r_{1}}+\frac{a_{2}^{2}}{r_{2}}+\frac{a_{3}^{2}}{r_{3}}+\frac{a_{4}^{2}}{r_{4}}\right]^{-1}, \\
& u_{3}=\frac{100 a_{3}}{r_{3} N}\left[\frac{a_{1}^{2}}{r_{1}}+\frac{a_{2}^{2}}{r_{2}}+\frac{a_{3}^{2}}{r_{3}}+\frac{a_{4}^{2}}{r_{4}}\right]^{-1}, \\
& u_{4}=\frac{100 a_{4}}{r_{4} N}\left[\frac{a_{1}^{2}}{r_{1}}+\frac{a_{2}^{2}}{r_{2}}+\frac{a_{3}^{2}}{r_{3}}+\frac{a_{4}^{2}}{r_{4}}\right]^{-1} .
\end{aligned}
$$

Now putting back these values into $J_{\mathrm{LP}}$, we have

$$
J_{\mathrm{LP}}=100 \frac{p_{1} a_{1} r_{2} r_{3} r_{4}+p_{2} a_{2} r_{1} r_{3} r_{4}+p_{3} a_{3} r_{1} r_{2} r_{4}+p_{4} a_{4} r_{1} r_{2} r_{3}}{a_{1}^{2} r_{2} r_{3} r_{4}+a_{2}^{2} r_{1} r_{3} r_{4}+a_{3}^{2} r_{1} r_{2} r_{4}+a_{4}^{2} r_{1} r_{2} r_{3}} .
$$

We make two observations.

(i) If $r_{1}, r_{2}, r_{3}, r_{4} \geq 0$, then the two sets of weights $\left(r_{1}, r_{2}, r_{3}, r_{4}\right)$ and $\alpha\left(r_{1}, r_{2}, r_{3}, r_{4}\right)$ would produce the identical LQR optimal input sequences for any $\alpha>0$.

(ii) Practically, the usage of electric energy is always positive which implies $r_{1}^{*}>0$.

Based on these two observations, we may set $r_{1}=1$ and

$$
\begin{aligned}
& r_{1}=1, \quad r_{2}=r_{2} r_{1}=r_{2}, \\
& r_{3}=r_{3} r_{1}=r_{3}, \quad r_{4}=r_{4} r_{1}=r_{4}
\end{aligned}
$$

which in turn gives rise to

$$
J_{\mathrm{LP}}=100 \frac{p_{1} a_{1} r_{2} r_{3} r_{4}+p_{2} a_{2} r_{3} r_{4}+p_{3} a_{3} r_{2} r_{4}+p_{4} a_{4} r_{2} r_{3}}{a_{1}^{2} r_{2} r_{3} r_{4}+a_{2}^{2} r_{3} r_{4}+a_{3}^{2} r_{2} r_{4}+a_{4}^{2} r_{2} r_{3}} .
$$

Suppose $r_{i}^{*}$ 's minimize the above $J_{\mathrm{LP}}$. Then, the optimal $u_{i}$ 's are those in (28) by replacing $r_{i}$ 's by $r_{i}^{*}$ 's. These are the solutions without the inequality constraints (9). With (9), the best $r_{i}$ 's are those that minimize $J_{\mathrm{LP}}$ under the constraints that the resultant $u_{i}$ 's are in the box defined by the inequality constraints (9).

To support the above theoretical derivation, a large number of actual production data sets (over 2 thousands) are collected. Based on 30 randomly chosen data sets, we first solve the linear programming problem (2) and (4) directly using the command "linprog" embedded in MATLAB. We then solve the same problem by finding the optimal $r_{i}^{*}$ 's and the corresponding optimal input sequences $u_{i}^{*}[k]$ 's through the LQR optimization as discussed before. In each case, the two optimization results are not identical but very close about $1-2 \%$ difference in the cost functions. We believe that this small numerical difference of $1-2 \%$ is due to numerical imperfection of MATLAB for a large dimensional linear programming (8400 variables and 14000 constraints). 


\section{Parameter Estimation}

In the previous sections, the entries of the matrix $B$ are assumed to be available. In reality, $B$ depends on the melting process and in particular the load weight of scraps. In general, the entries of $B$ have to be estimated. To put parameter estimation into a perspective, we first describe the melting process. The melting process is a batch process. One batch of steel is called a heat. The time between taps is the amount of time required to produce a batch of liquid steel. Produced tons is measured using charged scrap tons. First, scrap is brought into the melt shop and an overhead crane is used to load the scrap into a charging bucket. When the charge bucket has been loaded, the loading crane picks up the charge bucket and places the scrap into the EAF. The EAF is typically operated utilizing a 3-charge practice and a melting cycle is composed of the following stages.

(1) Furnace Turn Around-the EAF is inspected and prepared for the next heat.

(2) Charge-the first scrap is put into the EAF.

(3) Melting-power is turned on and the scrap is melted until the volume is sufficiently reduced to fit the second charge into the EAF.

(4) Charge and melting cycle is repeated for the second and third scrap charges.

(5) Refine-alloys are added to the liquid steel as necessary and steel is $100 \%$ melted.

(6) Tap-the liquid steel is transferred from the EAF to a ladle for casting.

Obviously depending on the weight of scrap in three charges, the entry values of $B$ vary. To this end, we provide an identifier, $\mathrm{l}=$ low, $\mathrm{m}=$ medium, and $\mathrm{h}=$ high to each charge to reflect the amount of scrap loaded in each charge.

Therefore, there are totally 9 different scrap loading scenarios as shown in Table 2. For instance, if three charges for a particular heat are 32 tons (1st charge), 32 tons (2nd charge), and 18 tons (3rd charge), respectively, then it is identified as $(11,21,31)$. If three charges are 32 tons, 34 tons, and 23 tons, it is identified as $(11,2 \mathrm{~m}, 3 \mathrm{~h})$. For each scenario, the entries of $B$ are estimated separately. For actual estimation, let

$$
\Delta x[k]=x[k+1]-x[k] .
$$

Then, we have for $k=1,2, \ldots, N$,

$$
\begin{gathered}
\Delta x_{1}[k]=u_{1}[k] \varphi_{i}, \quad \Delta x_{2}[k]=u_{1}[k] \phi_{1}, \\
\Delta x_{3}[k]=\left(u_{1}[k], u_{2}[k], u_{3}[k], u_{4}[k]\right)\left(\begin{array}{l}
a_{1} \\
a_{2} \\
a_{3} \\
a_{4}
\end{array}\right) .
\end{gathered}
$$

TABLE 2: Algorithm for choosing charge identifiers based on charge weights.

\begin{tabular}{lccc}
\hline \multirow{2}{*}{ Charge number } & \multicolumn{3}{c}{ CW (charge weight, in tons) } \\
& 1 (low) & m (medium) & h (high) \\
\hline 1 & $\mathrm{CW}<33$ & $33 \leq \mathrm{CW} \leq 35$ & $\mathrm{CW}>35$ \\
2 & $\mathrm{CW}<33$ & $33 \leq \mathrm{CW} \leq 35$ & $\mathrm{CW}>35$ \\
3 & $\mathrm{CW}<19$ & $19 \leq \mathrm{CW} \leq 22$ & $\mathrm{CW}>22$ \\
\hline
\end{tabular}

The estimates $\widehat{\varphi}_{1}, \widehat{\phi}_{1}$, and $\widehat{a}_{i}, i=1,2,3,4$, of $\varphi_{1}, \phi_{1}, a_{i}$ defined as solutions of the least squares fits

$$
\begin{aligned}
& \widehat{\varphi}_{1}=\arg \min _{\widehat{\varphi}} \sum_{k=1}^{N}\left(\Delta x_{1}[k]-u_{1}[k] \widehat{\varphi}\right)^{2}, \\
& \widehat{\phi}_{1}=\arg \min _{\widehat{\phi}} \sum_{k=1}^{N}\left(\Delta x_{2}[k]-u_{1}[k] \widehat{\phi}\right)^{2}, \\
& \left(\widehat{a}_{1}, \widehat{a}_{2}, \widehat{a}_{3}, \widehat{a}_{4}\right) \\
& =\arg \min _{\widehat{a}_{i}} \sum_{k=1}^{N}\left(\begin{array}{c}
\Delta x_{3}[k]-\left(u_{1}[k], u_{2}[k], u_{3}[k], u_{4}[k]\right) \\
\left.\times\left(\begin{array}{c}
\widehat{a}_{1} \\
\widehat{a}_{2} \\
\widehat{a}_{3} \\
\widehat{a}_{4}
\end{array}\right)\right)^{2},
\end{array}\right.
\end{aligned}
$$

can be readily solved as

$$
\begin{aligned}
& \widehat{\varphi}_{1}=\frac{\sum_{k=1}^{N} \Delta x_{1}[k] u_{1}[k]}{\sum_{k=1}^{N} u_{1}[k]^{2}}, \\
& \widehat{\phi}_{1}=\frac{\sum_{k=1}^{N} \Delta x_{2}[k] u_{1}[k]}{\sum_{k=1}^{N} u_{1}[k]^{2}}, \\
& \left(\begin{array}{l}
\widehat{a}_{1} \\
\widehat{a}_{2} \\
\widehat{a}_{3} \\
\widehat{a}_{4}
\end{array}\right)=\left(\sum_{k=1}^{N} u[k] u^{T}[k]\right)^{-1} \sum_{k=1}^{N} u[k] \Delta x_{3}[k] .
\end{aligned}
$$

To validate the parameter estimation results, we let

$$
\begin{aligned}
& \widehat{\Delta x}_{1}[k]=u_{1}[k] \widehat{\varphi}_{1}, \quad \widehat{\Delta x}_{2}[k]=u_{1}[k] \widehat{\phi}_{1}, \\
& \widehat{\Delta x}_{3}[k]=u^{T}[k]\left(\begin{array}{c}
\widehat{a}_{1} \\
\widehat{a}_{2} \\
\widehat{a}_{3} \\
\widehat{a}_{4}
\end{array}\right)
\end{aligned}
$$

and define the Goodness-of Fit as

$$
\begin{array}{r}
\mathrm{GoF}_{i}=1-\sqrt{\sum_{k=1}^{N} \frac{\left(\Delta x_{i}[k]-\widehat{\Delta x}_{i}[k]\right)^{2}}{\left(\Delta x_{i}[k]-(1 / N) \sum_{m=1}^{N} \Delta x_{i}[m]\right)^{2}}}, \\
i=1,2,3 .
\end{array}
$$


TABLE 3: GoFs.

\begin{tabular}{lccc}
\hline & $\begin{array}{c}\text { GoF of } \Delta x_{1} \\
(\text { KWH })\end{array}$ & $\begin{array}{c}\text { GoF of } \Delta x_{2} \\
\left(I^{2} h\right)\end{array}$ & $\begin{array}{c}\text { GoF of } \Delta x_{3} \\
(\mathrm{PM})\end{array}$ \\
\hline Max GoF & 0.9992 & 0.9995 & 0.9948 \\
Min GoF & 0.9984 & 0.9992 & 0.9930 \\
Avg GoF & 0.9990 & 0.9994 & 0.9941 \\
\hline
\end{tabular}

TABle 4: Production cost reduction of 15 heat files.

\begin{tabular}{lcc}
\hline $\begin{array}{l}\text { Heat file } \\
\text { number }\end{array}$ & $\begin{array}{c}\text { Ratio of the costs by } \\
\text { optimal control and } \\
\text { manual control }\end{array}$ & Cost saving \\
\hline 1 & 0.86 & $14 \%$ \\
2 & 0.9 & $10 \%$ \\
3 & 0.9 & $10 \%$ \\
4 & 0.9 & $10 \%$ \\
5 & 0.9 & $10 \%$ \\
6 & 0.86 & $14 \%$ \\
7 & 0.93 & $7 \%$ \\
8 & 0.84 & $16 \%$ \\
9 & 0.87 & $13 \%$ \\
10 & 0.91 & $9 \%$ \\
11 & 0.78 & $22 \%$ \\
12 & 0.9 & $10 \%$ \\
13 & 0.81 & $19 \%$ \\
14 & 0.82 & $18 \%$ \\
15 & 0.84 & $16 \%$ \\
\hline
\end{tabular}

The parameters are derived from one heat file and validated by 30 other fresh heat files, which were not used for estimation but with the same identifier. The validation results are shown in Table 3.

Clearly from Table 3 , the results are satisfactory and the values of $\widehat{\varphi}_{1}, \widehat{\phi}_{1}$, and $\widehat{a}_{i}$ will be used in the optimal control design to replace the unknown $\varphi_{1}, \phi_{1}$, and $a_{i}$ 's.

\section{Control Design Validation}

As discussed before, the control design involves two steps: (1) estimate the entries of $B$ for different scrap load weights and (2) based on the estimates calculate the optimal $r_{i}$ for the LQR problem and the corresponding optimal inputs $u_{i}^{*}\left(r_{1}^{*}, r_{2}^{*}, r_{3}^{*}, r_{4}^{*}\right)$. Recall that our goal is to design optimal input sequences so that the production cost (2) is minimized as compared to the current manual operation in which experiences play a key role. In theory, it has been demonstrated that the LQR solution $u_{i}^{*}\left(r_{1}^{*}, r_{2}^{*}, r_{3}^{*}, r_{4}^{*}\right), i=1,2,3,4$, solves the LP problem and consequently minimizes the production cost (2) under the constraints. Whether it works in reality needs to be validated. To this end, we randomly choose 15 actual heat files with known production costs. For each heat file, a fresh heat file with the same identifier but not in the original 15 is chosen and used to estimate the entries of $B$ to build the EAF model and calculate the optimal LQR solutions $u_{i}^{*}\left(r_{1}^{*}, r_{2}^{*}, r_{3}^{*}, r_{4}^{*}\right), i=1,2,3,4$. The optimal inputs are applied back to the original file to compute the production cost by the optimal control design. Based on the original production cost (2), the ratios of the costs by the optimal control designs derived by the LQR in which the weights $r_{i}$ 's are adjusted to minimize the LP cost and by the current manual practice are shown in Table 4 along with their percentage of cost saving. In all 15 experiments (files), the optimal inputs meet the constraints (9) and reduce the production cost ranging from $7 \%$ to $22 \%$. This supports our theoretical derivation and demonstrates that the production cost in which the energy consumption is a main part can be reduced substantially by changing the practice from manual to automatic.

\section{Conclusion and Future Work}

The method proposed for the optimal control strategy of an arc furnace provides a closed loop robust scheme that optimizes the production cost of the EAF steel making. In this study, we focus on 7 key variables and a linear modeling. In the future, other minor variables and possibly nonlinear effects could and should be considered. It is expected that by combining all the factors, the production cost can be reduced further. Also, the strategy developed in this paper can be extended to other applications in which the cost function is linear and the control design used is quadratic.

\section{Conflict of Interests}

The author declares that there is no conflict of interests regarding the publication of this paper.

\section{Acknowledgment}

This work was supported in part by the Iowa Energy Center Grant 09-01.

\section{References}

[1] R. Balan, V. Maties, O. Hancu, S. Stan, and L. Ciprian, "Modeling and control of an electric arc furnace," in Proceedings of the 15 Mediterranean Conference on Control and Automation, pp. 16, Athens, Greece, July 2007.

[2] B. Boulet, G. Lalli, and M. Ajersch, "Modeling and control of an electric arc furnace," in Proceedings of the American Control Conference, pp. 3060-3064, Denver, Colo, USA, June 2003.

[3] Ü. Çamdali and M. Tunç, "Modelling of electric energy consumption in the AC electric arc furnace," International Journal of Energy Research, vol. 26, no. 10, pp. 935-947, 2002.

[4] Anuradha, K. B. Muni, and A. Kumar, "Modeling of EFA and control algorithms for voltage flicker mitigation using DSTATCOM," in Proceedings of the 16th National Power Systems Conference, Hyderabad, India, December 2010.

[5] M. Han and X. Huang, "Greedy kernel components acting on ANFIS to predict BOF steelmaking endpoint," in Proceedings of the 17th World Congress, International Federation of Automatic Control (IFAC '08), Seoul, South Korea, July 2008. 
[6] L. Hocine, D. Yacine, K. M. Samira, and B. Kamel, "Closely parametrical model for an electrical arc furnace," World Academy of Science, Engineering and Technology, vol. 40, pp. 96-100, 2008.

[7] F. Lewis and V. Syrmos, Optimal Control, John Wiley \& Sons, New York, NY, USA, 1995.

[8] K. Zhou, J. Doyle, and K. Glover, Robust and Optimal Control, Prentice Hall, Upper Saddle River, NJ, USA, 1995. 


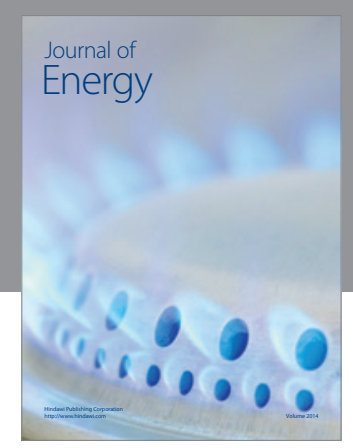

Journal of

Industrial Engineering
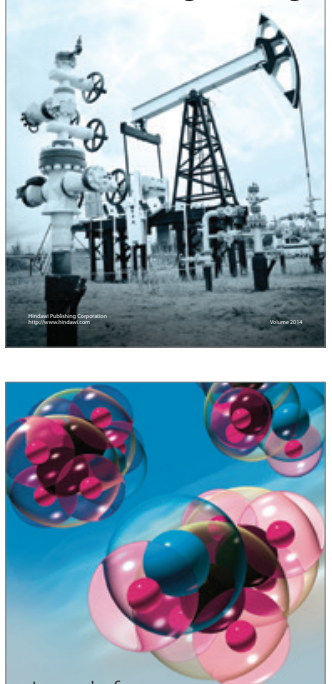

Fuels
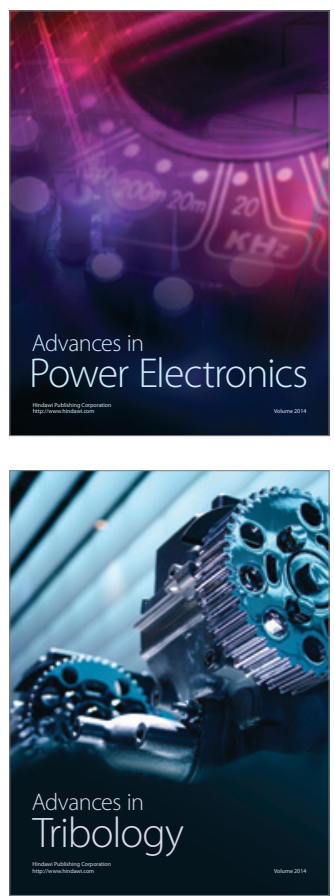

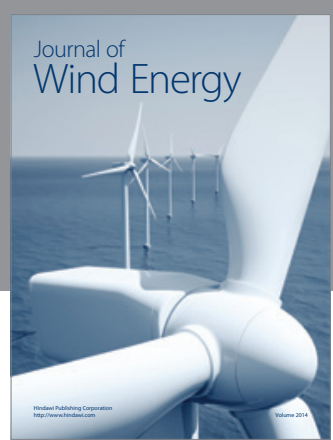

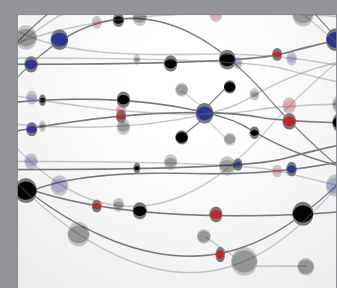

The Scientific World Journal

Submit your manuscripts at http://www.hindawi.com

Journal of

Structures
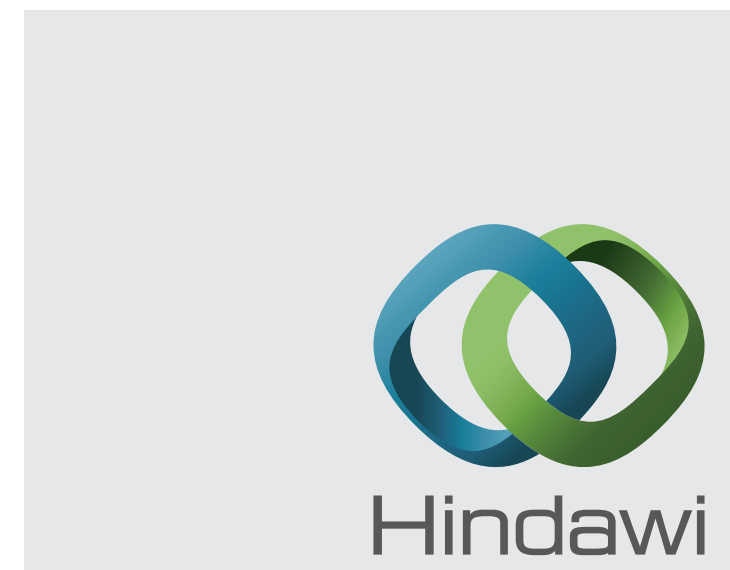

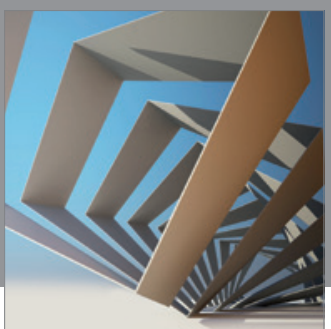

Rotating

Machinery
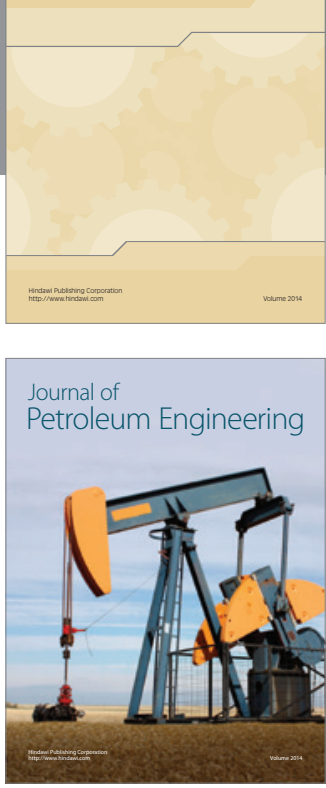

Journal of

Solar Energy
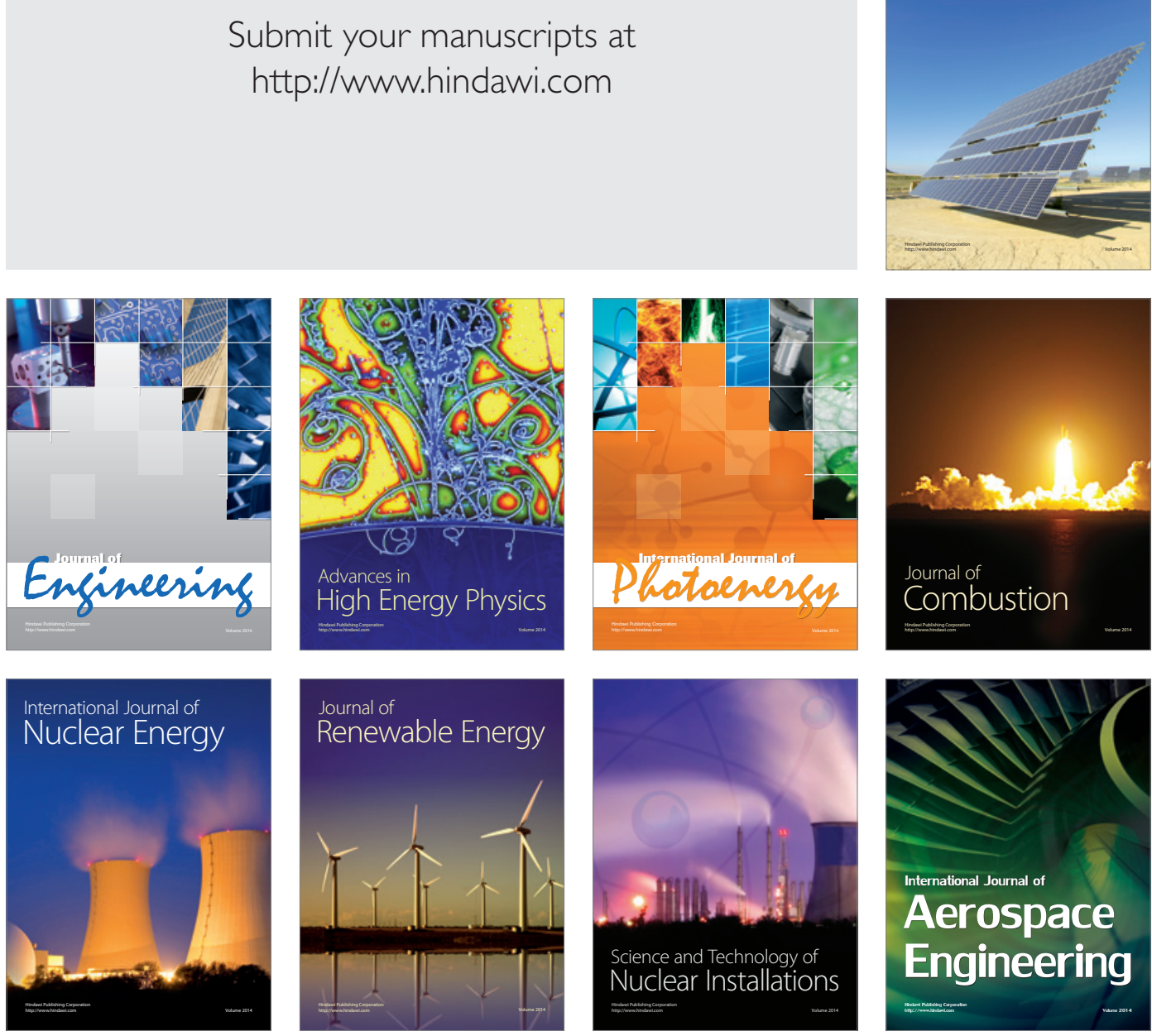